\title{
PRE-FRAME OPERATORS, BESSELIAN FRAMES, AND NEAR-RIESZ BASES IN HILBERT SPACES
}

\author{
JAMES R. HOLUB
}

(Communicated by Dale Alspach)

\begin{abstract}
A problem of enduring interest in connection with the study of frames in Hilbert space is that of characterizing those frames which can essentially be regarded as Riesz bases for computational purposes or which have certain desirable properties of Riesz bases. In this paper we study several aspects of this problem using the notion of a pre-frame operator and a model theory for frames derived from this notion. In particular, we show that the deletion of a finite set of vectors from a frame $\left\{x_{n}\right\}_{n=1}^{\infty}$ leaves a Riesz basis if and only if the frame is Besselian (i.e., $\sum_{n=1}^{\infty} a_{n} x_{n}$ converges $\Leftrightarrow\left(a_{n}\right) \in l^{2}$ ).
\end{abstract}

\section{INTRODUCTION}

Let $H$ denote a separable, infinite-dimensional Hilbert space. A sequence $\left\{x_{n}\right\}_{n=1}^{\infty}$ in $H$ is called a frame [3] if there exist positive numbers $A$ and $B$ for which

$$
A\|x\|^{2} \leq \sum_{n=1}^{\infty}\left|\left(x, x_{n}\right)\right|^{2} \leq B\|x\|^{2}
$$

for all $x$ in $H$. Of course, a particular example of a frame (and one that provides the motivation for the entire theory) is an orthonormal basis for $H$ or, more generally, any isomorphic image of an orthonormal basis, i.e., a Riesz basis [6, p. 160] for $H$.

Generally speaking, frames provide a natural generalization of orthonormal bases in problems involving the expansion of vectors in $H$ into a series in situations where uniqueness of representation may be unimportant and where an attempt to use an orthonormal basis may actually be a hindrance to effective computation (see [5] and its references). In the particular case where $H=L^{2}(\mu)$, certain frames are called wavelets (e.g., [5]) and have become an important tool for handling such expansion problems in cases where Fourier series methods are difficult to apply (see, e.g., $[1,2,7,12]$ ).

As these remarks suggest, the heart of the notion of a frame $\left\{x_{n}\right\}_{n=1}^{\infty}$ lies in the possibility of expanding vectors in $H$ as a convergent series $\sum_{n=1}^{\infty} a_{n} x_{n}$. In fact, every $x$ in $H$ has a "canonical" representation $x=\sum_{n=1}^{\infty}\left(S^{-1} x_{n}, x\right) x_{n}$,

Received by the editors July 21, 1992 and, in revised form, February 17, 1993.

1991 Mathematics Subject Classification. Primary 46B15, 46C05; Secondary 47A53.

Key words and phrases. Frame, Riesz basis, Fredholm operator. 
where $S$ is the so-called frame operator associated with $\left\{x_{n}\right\}_{n=1}^{\infty}$ [5], and hence one in which the coefficients of the series are in $l^{2}$. Except in the particular case where $\left\{x_{n}\right\}_{n=1}^{\infty}$ is a Riesz basis for $H$, a vector $x$ in $H$ will generally have infinitely many series representations in terms of $\left\{x_{n}\right\}_{n=1}^{\infty}$ and the coefficients in such expansions need not come out of $l^{2}$ (for example, let $\left\{x_{n}\right\}_{n=1}^{\infty}$ be the frame $\left\{e_{1},-e_{1}, e_{2},-e_{2}, \ldots\right\}$, so $x=\sum_{n=1}^{\infty} \frac{1}{n} e_{n}=\sum_{n=1}^{\infty} a_{n} x_{n}$ for $a_{2 n-1}=\frac{1}{n}+\frac{1}{\sqrt{n}}$ and $a_{2 n}=\frac{1}{\sqrt{n}}$ for all $n$ ).

A natural and important problem which arises, then, is that of determining when a frame is actually a Riesz basis for $H$ or, more generally, when a frame is a "near-Riesz basis" in the sense that the deletion of a finite subset leaves a Riesz basis (in the language of some authors, the frame has "finite excess"). The answer to the first of these questions is well known [5]; a frame $\left\{x_{n}\right\}$ is a Riesz basis $\Leftrightarrow$ it is exact (i.e., no vector in the set may be deleted without leaving an incomplete set). In this paper we study the second, more general, problem of determining when a frame is a near-Riesz basis. Central to this investigation is the introduction of what we call a "pre-frame operator" and the connection of this concept to that of a "Besselian frame", one whose convergent series expansions have coefficients in $l^{2}$. In particular, we show (Theorem 2.5) that a frame is a near-Riesz basis $\Leftrightarrow$ it is Besselian. In the process we develop a simple model theory for frames in which the study of abstract frames can be reduced to the study of a particular canonical type of frame whose properties are often more easily deduced.

The author expresses his appreciation to Professor C. Heil for his helpful comments regarding frames and their relationship to bases. The interested reader should consult the papers $[5,6]$, in particular, for the motivation and background for much of what appears here.

\section{BESSELIAN FRAMES AND NEAR-RIESZ BASES}

As usual, we denote by $l^{2}$ the Hilbert space of all square-summable sequences of scalars and by $\left\{e_{n}\right\}_{n=1}^{\infty}$ the unit vector basis for $l^{2}$ defined by $e_{n}=\left\{\delta_{i n}\right\}_{i=1}^{\infty}$. By analogy to the case of a Schauder basis (e.g., $[11$, pp. 337, 396]) we say that a frame $\left\{x_{n}\right\}_{n=1}^{\infty}$ for $H$ is:

(i) Besselian, if whenever $\sum_{n=1}^{\infty} a_{n} x_{n}$ converges, then $\left\{a_{n}\right\}_{n=1}^{\infty} \in l^{2}$;

(ii) unconditional, if whenever $\sum_{n=1}^{\infty} a_{n} x_{n}$ converges, it converges unconditionally; and

(iii) a near-Riesz basis, if there is a finite set $\sigma$ for which $\left\{x_{n}\right\}_{n \neq \sigma}$ is a Riesz basis for $H$.

As mentioned in $\S 1$, we begin our study with a simple characterization of frames in a Hilbert space as images of an orthonormal basis under a quotient map. In this context the relationship of general frames to Riesz bases becomes very transparent, as does the sense in which frames are a natural generalization of Riesz basis.

Theorem 2.1. A sequence of vectors $\left\{x_{n}\right\}_{n=1}^{\infty}$ in a Hilbert space $H$ is a frame $\Leftrightarrow$ there exists a bounded linear operator $Q$ from $l^{2}$ onto $H$ for which $Q e_{n}=x_{n}$ for all $n$.

Proof. $(\Rightarrow)$ If $\left\{x_{n}\right\}_{n=1}^{\infty}$ is a frame in $H$ then, by definition, there are positive constants $A$ and $B$ so that for all $x, A\|x\|^{2} \leq \sum_{n=1}^{\infty}\left|\left(x, x_{n}\right)\right|^{2} \leq B\|x\|^{2}$. 
In particular, $\sum_{n=1}^{\infty}\left|\left(x, x_{n}\right)\right|^{2}$ converges for all $x \in H$, so we can define a bounded linear operator $U: H \rightarrow l^{2}$ by $U(x)=\left\{\left(x, x_{n}\right)\right\}_{n=1}^{\infty}$. Clearly $\|U(x)\|^{2} \geq A\|x\|^{2}$, so $U$ is bounded below on the unit sphere in $H$ and hence has closed range. It follows that $Q=U^{*}$ maps $l^{2}$ onto $H[4$, p. 487], and since $\left(x, Q e_{n}\right)=\left(U x, e_{n}\right)=\left(x, x_{n}\right)$ for all $n$ and all $x \in H, Q e_{n}=x_{n}$ for all $n$.

$(\Leftarrow)$ On the other hand, suppose $Q$ is an operator from $l^{2}$ onto $H$ for which $Q e_{n}=x_{n}$ for all $n$. Then for any $x \in H, Q^{*}(x)=\sum_{n=1}^{\infty}\left(Q^{*} x, e_{n}\right) e_{n}=$ $\sum_{n=1}^{\infty}\left(x, x_{n}\right) e_{n}$. If we set $B=\left\|Q^{*}\right\|^{2}$, it follows that $B\|x\|^{2} \geq\left\|Q^{*} x\right\|^{2}=$ $\sum_{n=1}^{\infty}\left|\left(x, x_{n}\right)\right|^{2}$. Moreover, since $Q$ maps onto $H$, the operator $Q^{*}$ is one-toone with closed range [4, p. 487] and hence is bounded below on the unit sphere in $H$. That is, there is some positive number $A$ for which $\sum_{n=1}^{\infty}\left|\left(x, x_{n}\right)\right|^{2}=$ $\left\|Q^{*} x\right\|^{2} \geq A\|x\|^{2}$ for all $x \in H$. By definition, then, $\left\{x_{n}\right\}_{n=1}^{\infty}$ is a frame for $H$ and the theorem is proved.

Terminology. 1. If $\left\{x_{n}\right\}_{n=1}^{\infty}$ is a frame in $H$, the quotient map $Q: l^{2} \rightarrow H$ for which $Q e_{n}=x_{n}$ guaranteed by Theorem 2.1 is clearly unique and has the property that $Q Q^{*}(x)=\sum_{n=1}^{\infty}\left(x_{n}, x\right) x_{n}$ for all $x \in H$. That is, $Q Q^{*}$ is the so-called frame operator [5] associated with $\left\{x_{n}\right\}_{n=1}^{\infty}$; consequently, we call $Q$ the pre-frame operator associated with $\left\{x_{n}\right\}_{n=1}^{\infty}$.

2. As is customary for the case of bases in Banach spaces [11, p. 68] we will say that two frames $\left\{x_{n}\right\}_{n=1}^{\infty}$ and $\left\{y_{n}\right\}_{n=1}^{\infty}$ in Hilbert spaces $H_{1}$ and $H_{2}$, respectively, are equivalent if there is an isomorphism $V$ from $H_{1}$ onto $H_{2}$ for which $V x_{n}=y_{n}$ for all $n$. Clearly, then, equivalent frames have the same linear-topological properties and hence may be identified with one another.

Now if $P$ denotes the orthogonal projection of $l^{2}$ onto a closed subspace $X$ of $l^{2}$, by Theorem 2.1 the set $\left\{P e_{n}\right\}_{n=1}^{\infty}$ is a frame for the Hilbert space $X$. On the other hand, if $\left\{x_{n}\right\}$ is an arbitrary frame in $H$ then by Theorem 2.1 there is an operator $Q$ from $l^{2}$ onto $H$ for which $Q e_{n}=x_{n}$ for all $n$. It follows that if $P$ is the orthogonal projection of $l^{2}$ onto $(\operatorname{ker} Q)^{\perp}$ then $Q\left(P e_{n}\right)=x_{n}$ for all $n$ and $Q$ maps $(\operatorname{ker} Q)^{\perp}$ isomorphically onto $H$. That is, the frames $\left\{x_{n}\right\}_{n=1}^{\infty}$ in $H$ and $\left\{P e_{n}\right\}_{n=1}^{\infty}$ in $(\operatorname{ker} Q)^{\perp}$ are equivalent (under the induced isomorphism $Q$ ), and the study of arbitrary frames is reduced to the study of frames of the form $\left\{P e_{n}\right\}_{n=1}^{\infty}$ in a closed subspace of $l^{2}$.

We state this result formally for future reference (keeping in mind, as well, the details of the equivalence of the frames involved as outlined above):

Theorem 2.2. Every frame is equivalent to one of the form $\left\{P e_{n}\right\}_{n=1}^{\infty}$, where $P$ is some orthogonal projection on $l^{2}$.

As we remarked earlier, we will use these ideas to study the problem of characterizing frames which are near-Riesz bases. In particular, we examine the relationship between Besselian frames and Riesz bases in a Hilbert space. Obviously, a Besselian frame in $H$ need not actually be a Riesz basis since a frame, in general, need not even be linearly independent. For example, the frame $\left\{e_{1}, e_{1}, e_{2}, e_{3}, \ldots\right\}$ in $l^{2}$ is certainly Besselian but not a Riesz basis! However, this example and others like it turn out to be indicative of the general situation: If $\left\{x_{n}\right\}_{n=1}^{\infty}$ is a Besselian frame, then by deleting some finite number of vectors we are left with a Riesz basis. The following theorem on pre-frame operators forms the basis for establishing this result. 
Theorem 2.3. Let $\left\{x_{n}\right\}_{n=1}^{\infty}$ be a Besselian frame in $H$. If $Q: l^{2} \rightarrow H$ is the preframe operator associated with $\left\{x_{n}\right\}_{n=1}^{\infty}$, then $Q$ has finite-dimensional kernel.

Proof. Let $P$ denote the orthogonal projection of $l^{2}$ onto $(\operatorname{ker} Q)^{\perp}$. As we showed in Theorem 2.2, $\left\{P e_{n}\right\}_{n=1}^{\infty}$ is a frame in $(\operatorname{ker} Q)^{\perp}$ which is equivalent to $\left\{x_{n}\right\}_{n=1}^{\infty}$ and hence Besselian by assumption. Since $\operatorname{ker} Q=\operatorname{ker} P$, we need only show that $\operatorname{ker} P$ is finite dimensional.

Suppose, to the contrary, that $\operatorname{ker} P$ is infinite dimensional and therefore has an orthonormal basis $\left\{\phi_{i}\right\}_{i=1}^{\infty}$. Then $0=P \phi_{1}=\sum_{n=1}^{\infty}\left(e_{n}, \phi_{1}\right) P e_{n}$, so since $\left\|\phi_{1}\right\|=1$ we can choose some $N_{1}$ for which both $\left\|\sum_{n=1}^{N_{1}}\left(e_{n}, \phi_{1}\right) e_{n}\right\|>\frac{1}{\sqrt{2}}$ and $\left\|\sum_{n=1}^{N_{1}}\left(e_{n}, \phi_{1}\right) P e_{n}\right\|>\frac{1}{4}$. Since $\left\{\phi_{i}\right\}$ converges weakly to zero in $l^{2}$, we can then choose some $m_{2}>m_{1}=1$ for which $\left\|\phi_{m_{2}}-\sum_{n=N_{1}+1}^{\infty}\left(e_{n}, \phi_{m_{2}}\right) e_{n}\right\|<$ $\frac{1}{16}$. As in the previous step, it follows from this and the fact that $P \phi_{m_{2}}=$ 0 that we can get some $N_{2}>N_{1}$ so that $\left\|\sum_{n=N_{1}+1}^{N_{2}}\left(e_{n}, \phi_{m_{2}}\right) e_{n}\right\|>\frac{1}{\sqrt{2}}$ and $\left\|\sum_{n=N_{1}+1}^{N_{2}}\left(e_{n}, \phi_{m_{2}}\right) P e_{n}\right\|>\frac{1}{8}$. That is,

$$
\begin{aligned}
& \left\|\sum_{n=N_{1}+1}^{N_{2}} \frac{1}{\sqrt{2}}\left(e_{n}, \phi_{m_{2}}\right) e_{n}\right\|>\frac{1}{\sqrt{2} \cdot \sqrt{2}}, \\
& \left\|\sum_{n=N_{1}+1}^{N_{2}} \frac{1}{\sqrt{2}}\left(e_{n}, \phi_{m_{2}}\right) P e_{n}\right\|<\frac{1}{\sqrt{2} \cdot 8},
\end{aligned}
$$

and, of course,

$$
\left\|\sum_{n=N_{1}+1}^{N_{2}} \frac{1}{\sqrt{2}}\left(e_{n}, \phi_{m_{2}}\right) P e_{n}\right\| \leq \frac{1}{\sqrt{2}} \text { for all } r \geq N_{1}+1 .
$$

By the same argument, choose $m_{3}>m_{2}$ so $\left\|\phi_{m_{3}}-\sum_{n=N_{2}+1}^{\infty}\left(e_{n}, \phi_{m_{3}}\right) e_{n}\right\|<\frac{1}{32}$ and choose $N_{3}>N_{2}$ so that $\left\|\sum_{n=N_{2}+1}^{N_{3}} \frac{1}{\sqrt{3}}\left(e_{n}, \phi_{m_{3}}\right) e_{n}\right\|>\frac{1}{\sqrt{3} \cdot \sqrt{2}}$, while

$$
\left\|\sum_{n=N_{2}+1}^{N_{3}} \frac{1}{\sqrt{3}}\left(e_{n}, \phi_{m_{3}}\right) P e_{n}\right\|<\frac{1}{\sqrt{3}-16} \text { and }\left\|\sum_{n=N_{2}+1}^{r} \frac{1}{\sqrt{3}}\left(e_{n}, \phi_{m_{3}}\right) P e_{n}\right\| \leq \frac{1}{\sqrt{3}}
$$

for all $r \geq N_{2}+1$.

Continuing in this fashion we construct increasing sequences $\left\{m_{K}\right\}_{K=1}^{\infty}$ and $\left\{N_{K}\right\}_{K=0}^{\infty}\left(\right.$ where $\left.N_{0}=1\right)$ for which

$$
\begin{aligned}
& \left\|\sum_{n=N_{K}+1}^{N_{K+1}} \frac{1}{\sqrt{K+1}}\left(e_{n}, \phi_{m_{K+1}}\right) e_{n}\right\|>\frac{1}{\sqrt{K+1} \cdot \sqrt{2}}, \\
& \left\|\sum_{n=N_{K}+1}^{N_{K+1}} \frac{1}{\sqrt{K+1}}\left(e_{n}, \phi_{m_{K+1}}\right) P e_{n}\right\|<\frac{1}{\sqrt{K+1} \cdot 2^{K+2}},
\end{aligned}
$$

and

$$
\left\|\sum_{n=N_{K}+1}^{r} \frac{1}{\sqrt{K+1}}\left(e_{n}, \phi_{m_{K+1}}\right) P e_{n}\right\| \leq \frac{1}{\sqrt{K+1}} \text { for all } r \geq N_{K}+1
$$


Clearly, the series

$$
\sum_{n=1}^{\infty} c_{n} P e_{n}=\sum_{K=0}^{\infty} \sum_{n=N_{K}+1}^{N_{K+1}} \frac{1}{\sqrt{K+1}}\left(e_{n}, \phi_{m_{K+1}}\right) P e_{n}
$$

converges in $l^{2}$ (by (ii) and (iii)). However, by (i) (and the divergence of the harmonic series) it is also clear that $\sum_{n=1}^{\infty} c_{n}^{2}=+\infty$, so $\left\{P e_{n}\right\}_{n=1}^{\infty}$ cannot be a Besselian frame. But this is a contradiction to our assumption, and it follows that $\operatorname{ker} P$ must be finite dimensional.

Remark. Recall that an operator from one Banach space to another is called a Fredholm operator $[10$, p. 125$]$ if it is onto and has a finite-dimensional kernel. Using such terminology, Theorem 2.3 can be stated as follows: If $\left\{x_{n}\right\}_{n=1}^{\infty}$ is a Besselian frame in $H$, the associated pre-frame operator $Q$ is a Fredholm operator.

In fact, the converse to Theorem 2.3 is also true but is really only a simple consequence of our next result which shows that it is the finite-dimensionality of the kernel of the pre-frame operator which determines the Riesz basis-like behavior of the frame.

Theorem 2.4. Let $\left\{x_{n}\right\}_{n=1}^{\infty}$ be a frame in $H$ and $Q: l^{2} \rightarrow H$ the associated preframe operator. Then $\operatorname{ker} Q$ is finite dimensional $\Leftrightarrow\left\{x_{n}\right\}_{n=1}^{\infty}$ is a near-Riesz basis for $H$.

Proof. According to the comments preceding Theorem 2.2, if $P$ is the orthogonal projection of $l^{2}$ onto $(\operatorname{ker} Q)^{\perp}$ then $\left\{P e_{n}\right\}_{n=1}^{\infty}$ is a frame in $(\operatorname{ker} Q)^{\perp}$ which is equivalent to $\left\{x_{n}\right\}$. Hence, $\left\{x_{n}\right\}_{n=1}^{\infty}$ is a near-Riesz basis for $H$ if and only if $\left\{P e_{n}\right\}_{n=1}^{\infty}$ is a near-Riesz basis for $(\operatorname{ker} Q)^{\perp}=\operatorname{ran} P$.

Now suppose $\operatorname{ker} Q \quad(=\operatorname{ker} P)$ is finite dimensional. Then $I-P$ is the orthogonal projection onto this finite-dimensional subspace and hence is certainly a Hilbert-Schmidt operator [9, p. 34]. That is, $\sum_{n=1}^{\infty}\left\|(I-P) e_{n}\right\|^{2}<+\infty$, so there is an integer $N$ for which $\sum_{n=N+1}^{\infty}\left\|e_{n}-P e_{n}\right\|^{2}<1$. If we set $z_{n}=e_{n}$ for $n=1,2,3, \ldots, N$ and $z_{n}=P e_{n}$ for $n \geq N+1$, then $\sum_{n=1}^{\infty}\left\|e_{n}-z_{n}\right\|^{2}<1$, so $\left\{z_{n}\right\}_{n=1}^{\infty}$ is a basis for $l^{2}$ which is equivalent to $\left\{e_{n}\right\}_{n=1}^{\infty}$ [8]. In other words, $\left\{z_{n}\right\}_{n=1}^{\infty}$ is a Riesz basis for $l^{2}$, and it follows that $\left\{P e_{n}\right\}_{n=N+1}^{\infty}=\left\{z_{n}\right\}_{n=N+1}^{\infty}$ is a Riesz basis for its closed linear span $\left[P e_{n}\right]_{n=N+1}^{\infty}$ in $l^{2}$. But this is a subspace of finite codimension in $(\operatorname{ker} Q)^{\perp}$, and since $\left\{P e_{n}\right\}_{n=1}^{\infty}$ spans $(\operatorname{ker} Q)^{\perp}$ the set $\left\{P e_{n}\right\}_{n=N+1}^{\infty}$ can be extended by the addition of some subset of $\left\{P e_{n}\right\}_{n=1}^{N}$ to a (Riesz) basis for all of $(\operatorname{ker} Q)^{\perp}$. That is, there is a finite set $\sigma$ (in fact, a subset of $\{1,2, \ldots, N\})$ for which $\left\{P e_{n}\right\}_{n \notin \sigma}$ is a Riesz basis for $(\operatorname{ker} Q)^{\perp}$, and hence the corresponding set $\left\{x_{n}\right\}_{n \notin \sigma}$ is a Riesz basis for $H$.

Conversely, suppose $\left\{x_{n}\right\}_{n=1}^{\infty}$ is a near-Riesz basis for $H$, so there is some finite set $\sigma$ for which $\left\{x_{n}\right\}_{n \notin \sigma}$ is a Riesz basis for $H$. If $\sum_{n=1}^{\infty} a_{n} x_{n}$ converges then $\sum_{n \notin \sigma} a_{n} x_{n}$ certainly does, implying $\left\{a_{n}\right\}_{n \notin \sigma} \in l^{2}$. But then $\left\{a_{n}\right\}_{n=1}^{\infty} \in l^{2}$ also, and it follows that $\left\{x_{n}\right\}_{n=1}^{\infty}$ is a Besselian frame. By Theorem $2.3 \operatorname{ker} Q$ is finite dimensional, and the theorem is proved.

Putting Theorem 2.3 and 2.4 together with the observation that for any frame $\left\{x_{n}\right\}_{n=1}^{\infty}$, if $\left(a_{n}\right) \in l^{2}$ then $\sum_{n=1}^{\infty} a_{n} x_{n}$ converges, we get the following characterization of frames which are near-Riesz bases. 
Theorem 2.5. If $\left\{x_{n}\right\}_{n=1}^{\infty}$ is a frame in $H$, the following are equivalent:

(i) $\left\{x_{n}\right\}_{n=1}^{\infty}$ is a near-Riesz basis for $H$.

(ii) $\left\{x_{n}\right\}_{n=1}^{\infty}$ is Besselian.

(iii) $\sum_{n=1}^{\infty} a_{n} x_{n}$ converges in $H \Leftrightarrow\left\{a_{n}\right\}_{n=1}^{\infty} \in l^{2}$.

Note. The equivalence (i) $\Leftrightarrow$ (iii) of Theorem 2.5 is the direct analogue for frames of the well-known characterization of Riesz bases in a Hilbert space as those whose coefficient spaces coincide with $l^{2}[11$, p. 341].

\section{Related RESUlts}

Again, let $\left\{x_{n}\right\}_{n=1}^{\infty}$ be a frame in a Hilbert space $H$. According to Theorem 2.4 , there is some finite set $\sigma$ so that $\left\{x_{n}\right\}_{n \notin \sigma}$ is a Riesz basis for $H$ if and only if the pre-frame operator $Q$ associated with $\left\{x_{n}\right\}_{n=1}^{\infty}$ has a finite-dimensional kernel. We now give a more quantitative version of this result by showing that in this case the "excess" of the frame (i.e., the cardinality for the set $\sigma$ ) is actually equal to the dimension of the kernel of $Q$.

Theorem 3.1. Let $\left\{x_{n}\right\}_{n=1}^{\infty}$ be a frame in $H$ for which $\left\{x_{n}\right\}_{n} \notin \sigma$ is a Riesz basis for some finite set $\sigma$. If $Q$ denote the pre-frame operator associated with $\left\{x_{n}\right\}_{n=1}^{\infty}$, then $\operatorname{card} \sigma=\operatorname{dim}(\operatorname{ker} Q)$.

Proof. Suppose $\left\{x_{n}\right\}_{n \notin \sigma}$ is a Riesz basis for $H$, where $\sigma$ is some finite set. Let $P$ denote, as usual, the orthogonal projection of $l^{2}$ onto $(\operatorname{ker} Q)^{\perp}$ (so $\operatorname{ker} P=\operatorname{ker} Q)$. By Theorem 2.2 the set $\left\{P e_{n}\right\}_{n \notin \sigma}$ is a Riesz basis for $\operatorname{ran} P$ and $P$ acts as an isomorphism mapping $\left[e_{n}\right]_{n \notin \sigma}$ onto $\operatorname{ran} P$.

If $x \in l^{2}$, then $P x \in \operatorname{ran} P$, so there is a (unique) vector $\sum_{n \notin \sigma} a_{n} e_{n}$ in $\left[e_{n}\right]_{n \notin \sigma}$ for which $P\left(\sum_{n \notin \sigma} a_{n} x_{n}\right)=P x$ and hence for which $x-\sum_{n \notin \sigma} a_{n} x_{n} \in$ ker $P$. That is, $l^{2}=\operatorname{ker} P+\left[e_{n}\right]_{n \notin \sigma}$ (the algebraic sum of these subspaces). But if $z$ is in $\operatorname{ker} P \cap\left[e_{n}\right]_{n \notin \sigma}$, then $z=\sum_{n \notin \sigma} b_{n} e_{n}$ and $0=P z=\sum_{n \notin \sigma} b_{n} P e_{n}$, so since $\left\{P e_{n}\right\}_{n \notin \sigma}$ is a basis for $\operatorname{ran} P$, we see that $b_{n}=0$ for all $n$, and it follows that $z=0$. Therefore, $\operatorname{ker} P \cap\left[e_{n}\right]_{n \notin \sigma}=\{0\}$, and by the above we have that $l^{2}=\operatorname{ker} P \oplus\left[e_{n}\right]_{n \notin \sigma}$ (where this is a topological, but not necessarily orthogonal, $\operatorname{direct}$ sum $)$. But then $\operatorname{dim}(\operatorname{ker} P=\operatorname{card} \sigma$, and since $\operatorname{dim}(\operatorname{ker} Q)=\operatorname{dim}(\operatorname{ker} P)$, the theorem is proved.

Finally, we consider the relationship between the results of $\S 2$ and the notion of an unconditional frame introduced there.

Heil has shown [6, p. 168] that if $\left\{x_{n}\right\}_{n=1}^{\infty}$ is a frame in $H$ which is bounded below (i.e., $\inf _{n}\left\|x_{n}\right\|>0$ ) then (in our terminology) $\left\{x_{n}\right\}_{n=1}^{\infty}$ is Besselian $\Leftrightarrow\left\{x_{n}\right\}_{n=1}^{\infty}$ is unconditional. From this result and Theorem 2.5 we have the following characterization of unconditional frames:

Theorem 3.2. If $\left\{x_{n}\right\}_{n=1}^{\infty}$ is a frame in $H$ which is bounded below, then $\left\{x_{n}\right\}_{n=1}^{\infty}$ is unconditional $\Leftrightarrow\left\{x_{n}\right\}_{n=1}^{\infty}$ is a near-Riesz basis for $H$.

Again, Theorem 3.2 is the frame analogue of the corresponding well-known characterization of Riesz bases in Hilbert space as those which are bounded and unconditional [6, p. 160].

Remark. The notion of a pre-frame operator and the model theory for frames which it affords can also be applied with similar success to other problems involving frames, many of which are analogues of corresponding problems for bases. In a subsequent paper these ideas will be developed more fully. 


\section{REFERENCES}

1. I. Daubechies, Frames of coherent states, phase space localisation, and signal analysis (to appear).

2. I. Daubechies, A. Grossman, and Y. Meyer, Painless nonorthogonal expansions, J. Math. Phys. 27 (1986), 1271-1283.

3. R. Duffin and A. Schaeffer, A class of nonharmonic Fourier series, Trans. Amer. Math. Soc. 72 (1952), 341-366.

4. N. Dunford and J. Schwartz, Linear operators, I, Interscience, New York, 1963.

5. C. Heil, Wavelets and frames, Signal Processing, Part I, IMA Vol. Math. Appl., vol. 22, Springer-Verlag, Berlin, 1990, pp. 147-160.

6. C. Heil, Wiener amalgam spaces in generalized harmonic analysis and wavelet theory, $\mathrm{Ph} . \mathrm{D}$. Dissertation, University of Maryland, College Park, MD, 1990.

7. A. Janssen, Bargmann transform, Zak transform, and coherent states, J. Math. Phys. 23 (1982), 720-731.

8. J. Retherford and J. Holub, The stability of bases in Banach and Hilbert spaces, J. Reine Angew. Math. 246 (1971), 136-146.

9. J. Schatten, Norm ideals of completely continuous operators, Springer-Verlag, Berlin, 1970.

10. M. Schechter, Principles of functional analysis, Academic Press, New York, 1971.

11. I. Singer, Bases in Banach spaces. I, Springer-Verlag, Berlin, 1970.

12. J. Zak, Finite translations in solid state physics, Phys. Rev. Lett. 19 (1967), 1385-1397.

Department of Mathematics, Virginia Polytechnic Institute and State University, BLACKSBURG, VIRGINIA 24061

E-mail address: holubj@math.vt.edu 